\title{
Tissue Engineering for Bone Production- Stem Cells, Gene Therapy and Scaffolds
}

\author{
E.G. Khaled ${ }^{1}$, M. Saleh ${ }^{1}$, S. Hindocha ${ }^{* 2,3}$, M. Griffin ${ }^{3}$ and Wasim S. Khan ${ }^{4}$ \\ ${ }^{I}$ Department of Plastic Surgery, Ain Shams University, Plastic Surgery department, Ramsis Street, Abbassia Square, \\ Cairo, Postcode 11566, Egypt \\ ${ }^{2}$ Department of Plastic Surgery, Whiston Hospital, Warrington Road, Liverpool, L35 5DR, UK \\ ${ }^{3}$ University of Manchester. Manchester Interdisciplinary Biocentre, 131 Princess Street, Manchester, M1 7DN, UK \\ ${ }^{4}$ University College London Institute of Orthopaedics and Musculoskeletal Sciences, Royal National Orthopaedic \\ Hospital, Stanmore, Middlesex, HA7 4LP, UK
}

\begin{abstract}
A bone graft has been the gold standard treatment for repairing bone defects. However, due to bone grafts associated donor site morbidity several alternative bone substitutes options have been made available but with their added expense and limited osteoinductive properties they are not ideal. Therefore, research has begun in tissue engineering to investigate stem cells, which are one of the body's own mechanisms used to repair bone. Stem cells are clonogenic undifferentiated cells capable of self-renewal. Readily available from numerous of sources stem cells have the potential to differentiate in osteoblasts and chrondrocytes showing capability to repair both bone and cartilage. The known immunologic properties of stem cells further enhance their therapeutic appeal. Stem cells have shown to be excellent carriers for gene transfer having the capability to be transduced. Gene transfer could enable growth factors and bone morphogentic proteins to enhance bone repair. Stem cells are implanted onto scaffolds, which are structures capable of supporting tissue formation by allowing cell migration, proliferation and differentiation. Research aims to produce scaffolds that deliver and retain cells, allow for cell attachment has adequate biodegradability, biocompatibility and nonimmunogenicity. However, having tried and testing numerous materials including synthetic and natural products research into the perfect scaffold product continues. This review aims to explain how stem cells were discovered, the techniques used to isolate stem cells, identify and manipulate them down different cell lineages and discuss the research into using stem cells to reconstruct bone using genetic modification and scaffolds.
\end{abstract}

Keywords: Adult stem cells, bone marrow, bone reconstruction, gene therapy, scaffolds, tissue engineering.

Bone defects exceeding critical size usually heal with fibrous tissue (scar), missing complete bone re-union. The critical size is defined as intraosseous deficiency that will not heal with more than $10 \%$ new bone formation within life expectancy of the patient (human or nonhuman) [1].

Reconstruction of bone defects is dependent on certain mechanisms, which can be summarized into osteoconduction, osteoinduction and osteogenesis mechanisms. Osteogenesis is the formation of new bone from osteocompetent cells. Osteoconduction is the formation of bone along the scaffold of a biologic or alloplastic substance where the bone forming cells originate from pre-existing host osteocompetent cells. Osteoinduction is the formation of new bone by differentiation and stimulation of mesenchymal cells by bone inductive proteins [2].

In principle, bone grafts contain all the key elements required for bone repair; as they provide osteoconductive scaffold, growth factors for osteoinduction, and cells with osteogenic potential [3]. The use of bone grafts in the

\footnotetext{
*Address correspondence to this author at the Department of Plastic Surgery, Whiston Hospital, Warrington Road, Liverpool, L35 5DR, UK; Tel: 01244366265; Fax: 01244366277; E-mail: hindocha2001@yahoo.com
}

clinical practice presents several major inconveniences [4]. Although the percentage of success is high, complications as resorption, fracture, infection and non-union are still present [5]. Additionally harvesting of autologous bone often results in donor site morbidity, the extent of which may vary according to the location and the intervention technique including hemorrhage, nerve damage, cosmetic disability, pain, infection, and loss of function [6].

The use of human cadaver or animal bone grafts from bone banks prevents the problem of the donor site morbidity, but presents the potential risk of viral or bacterial infections and an immune response of the host tissue towards the implant. However, the continuous amelioration of the processing procedures for grafts is significantly reducing the risk of infection [7].

Alternatively, guided bone regeneration and several biomaterials have been considered and used as bone substitutes, including calcium phosphate ceramics, polymers and bioglasses. All have the advantage of unlimited availability and good osteoconductive properties. On the other hand, they are not osteoinductive, thus limiting their application to repair large bone defects [8]. On the contrary, bone distraction takes the advantage of bone regeneration potential; avoiding the troubles associated with the graft 
integration. However, it is highly problematic for the patients, technically demanding method and requires long interval to be completed [9].

Bone tissue engineering can guarantee unlimited source for bone regeneration without complications of other bone reconstruction modalities. Also, this technique is of great advantage in bone reconstruction in case of huge bone defects and in pediatric population; because of limited autogenous bone reservoir in addition to restricted use of alloplastic materials; because of ongoing skeletal growth [10].

\section{WHAT ARE STEM CELLS?}

The history of stem cells began in 1976, when certain cells were isolated and cultured, the product of this culture were adherent fibroblast like cells capable of proliferation and differentiation into bone and cartilage like colonies. These cells were given the name "colony forming unitfibroblast (CFU-F). Later on, these cells were named mesenchymal stem cells (1994), marrow stromal cells (1997) and mesenchymal progenitor cells (1999). Nowadays, the mesenchymal stem cell (MSC) is the most popular dominator given to these cells [3].

Stem cells are defined as clonogenic undifferentiated cells capable of self-renewal and differentiation into one or more cell progenies. Historically, adult MSCs were thought to be developmentally restricted to specific cell lineages that are related to the tissue in which the cells reside. Now, it is proven that the differentiation potential of adult MSCs is very versatile including all mesenchymal derived tissues. Moreover, under the appropriate conditions adult MSCs have the potential to transdifferentiate into cells possessing morphological and biochemical properties of ectodermal derived neural tissue, as well as dedifferentiation into pluripotent stem cell. The transdifferentiation property of adult MSCs was certified through detection of a myriad of neural markers in vitro, including nestin, mitogen-activated protein 2, Tau, NeuN, and glial fibrillary acidic protein [3].

To date, stem cells have been isolated and characterized from embryonic, fetal, and adult tissues. Embryonic stem (ES) cells are pluripotent, and are capable of unlimited proliferation in an undifferentiated state. Embryonic stem cells are totally uncommitted cells which need certain cell programming to be directed into specific cell progeny. The fetal stem cells are an intermediate cell type between ES and the adult stem cells. Adult stem cells are highly committed cells which need some reprogramming to differentiate into mature tissues [11].

Adult stem cells are the cornerstone stem cell model used in recent research; as it minimize the ethical and legal debates. Adult stem cells are all postnatal stem cells which can be classified into hematopoietic stem cells, epithelial stem cells and MSCs. Unlike the two other sources of adult stem cells; adult MSCs are more superior as they can be isolated from multiple sources like bone marrow, adipose tissue, periosteum, muscle, dermis, synovial membranes, etc. Also adult MSCs are multipotent cells capable of differentiation into a variety of mature tissues [3].

\section{THE IDEAL SOURCE OF ADULT STEM CELLS}

Bone marrow is a major source for osteogenic cells capable of forming bones in vitro. However, cell isolation procedure from bone marrow occasionally causes some disadvantages in clinical practice. Bone marrow aspiration is potentially more invasive and painful procedure, and it increases a risk of morbidity and infection. In addition to bone marrow-derived MSCs, periosteal-derived cells are also multipotent and have the potential to differentiate into osteoblasts and chondrocytes. Periosteal-derived cells have several advantages in comparison with bone marrow-derived MSCs, such as a minimal invasion to obtain cells, and easy cell harvest. These periosteal-derived cells also retain the property to differentiate into osteoblasts after following enzyme treatment and cell expansion [12].

An ideal source of autologous stem cells would, therefore, be both easy to obtain, result in minimal patient discomfort, yet be capable of yielding cell numbers substantial enough to obviate extensive expansion in culture. Adipose tissue, like bone marrow, is derived from the embryonic mesoderm and contains a heterogenous stromal cell population. These similarities make the concept that a stem cell population can be isolated from human adipose tissue. Moreover, the disadvantages of traditional bone marrow harvesting procedures in addition to low numbers of MSCs upon processing which necessitate an ex vivo expansion step to obtain clinically significant cell numbers. Such a step is time consuming, expensive, and risks cell contamination and loss. All these points of comparison make the adipose derived stem cell better source for stem cells [13].

\section{STEM CELLS IDENTIFICATION}

Identification of stem cells is achieved through their morphology, phenotypic characteristics and their biological behavior. Morphologically, stem cells are spindle shaped cells with central large nucleus, multiple cytoplasmic processes usually project from the outer surface. To date, there is no specific phenotypic marker to identify stem cells. However, stem cells are capable of expressing a lot of surface markers, these markers have also been detected in mesenchymal, endothelial, or epithelial cells. In conclusion, the stem cells can only be identified with their biological behavior as cells capable of proliferation and differentiation into different cell progenies [13].

Being one of the most popular sources of stem cells, research activities focused on the identification of bone marrow derived adult MSCs through three markers CD34, CD45, and CD90. CD34 is a surface marker of hematopoietic progenitors, CD45 is a lymphocytic surface marker, and CD90 is the major surface marker of mesenchymal cells [14]. We have previously shown that passage 2 synovial fat pad derived MSCs and bone marrow derived MSCs stained strongly for CD13, CD29, CD44, CD90 and CD105, and poorly for LNGFR, STRO1, CD34 and CD56 (Fig. 1). Occasional cells stained positively for $3 \mathrm{G} 5$ from both the synovial fat pad and bone marrow.

Although the molecular mechanisms governing MSC differentiation are not completely understood, a number of 


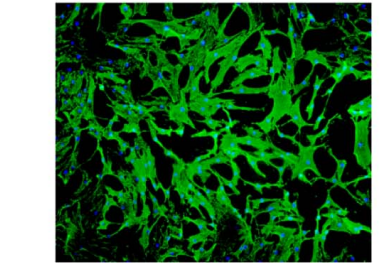

CD13
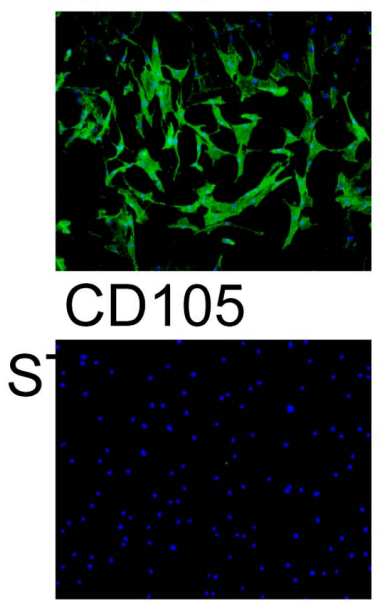

CD34

a

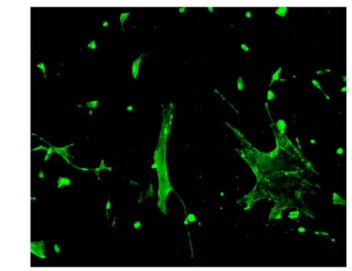

CD13

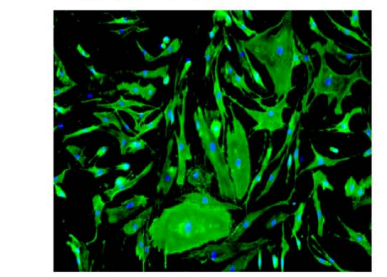

CD105

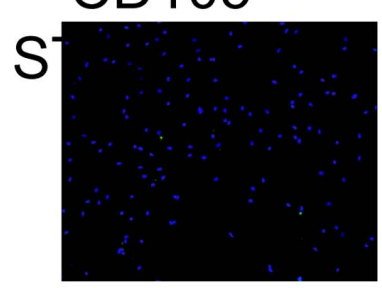

CD34

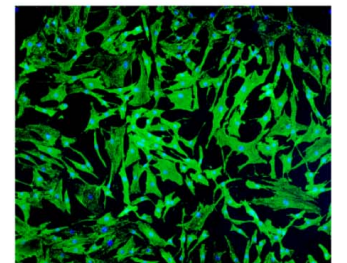

CD29

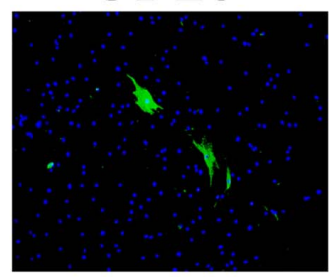

$3 G 5$

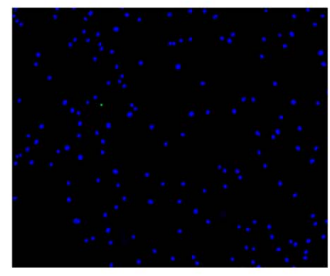

CD56

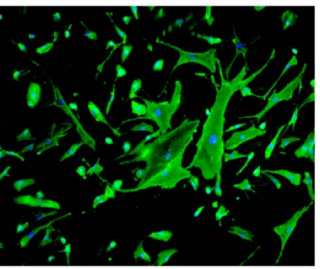

CD29

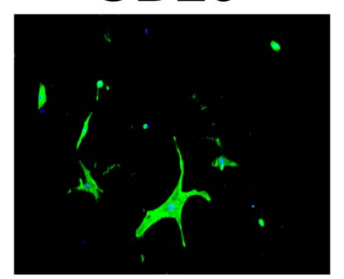

$3 G 5$

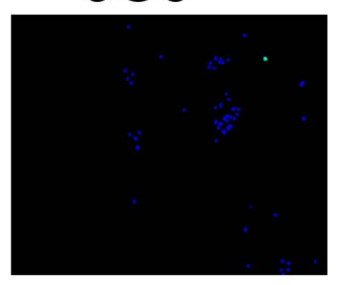

CD56

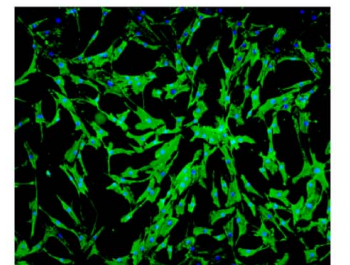

CD44

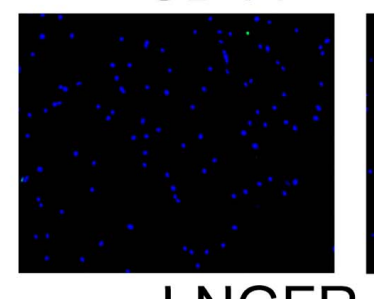

LNGFR

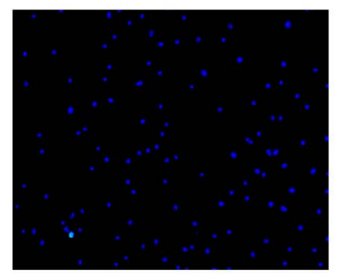

IgG control

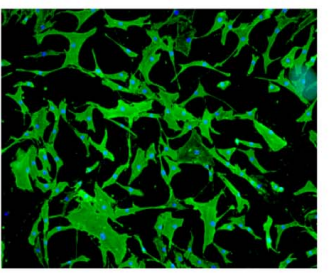

CD90
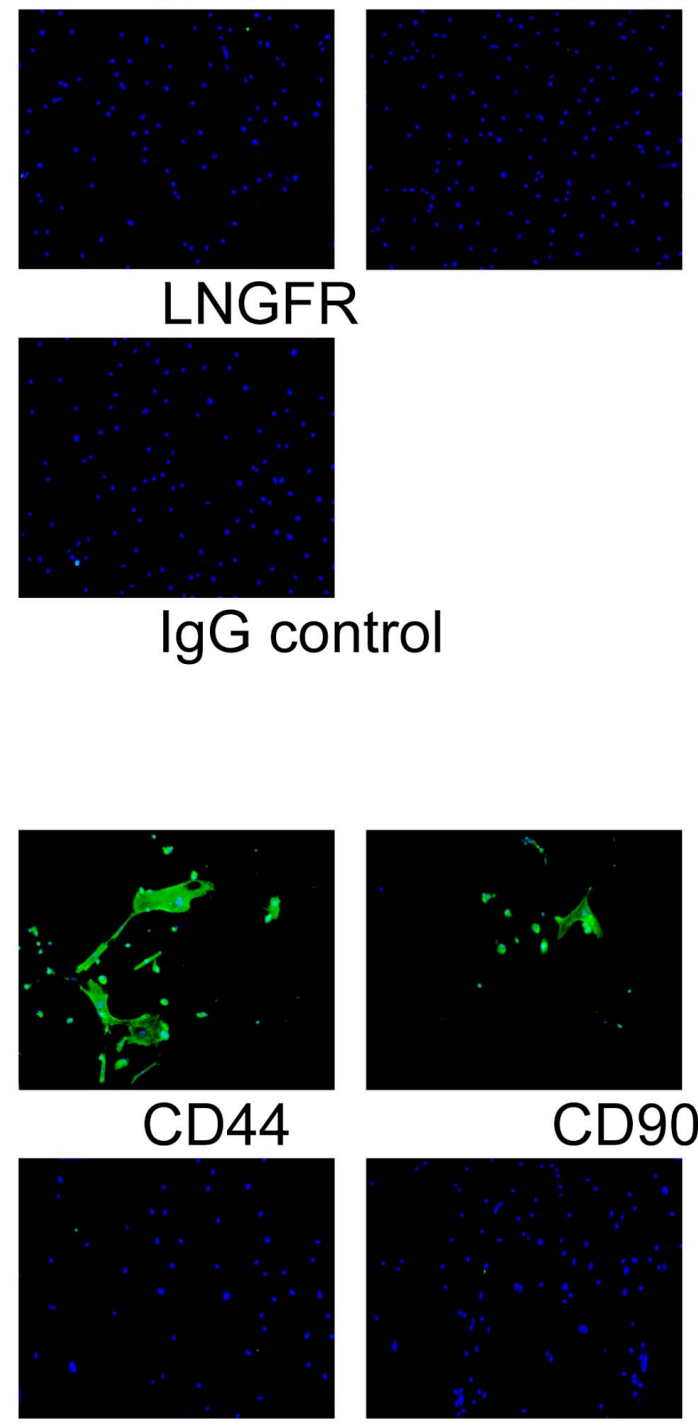

CD90

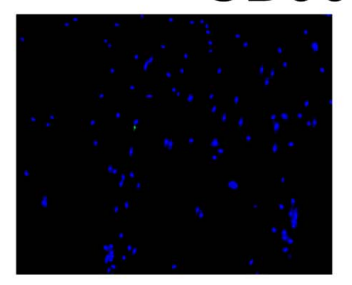

LNGFR

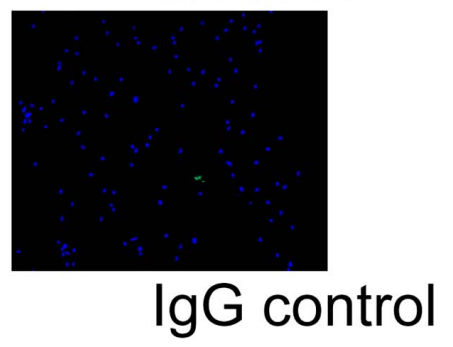

b

Fig. (1). Cell surface epitope characterisation of passage 2 synovial fat pad derived MSCs (a) and bone marrow derived MSCs (b) using a panel of antibodies. Cell surface staining using FITC conjugated secondary antibody (green) and DAPI (blue) shows that the cells stained strongly for CD13, CD29, CD44, CD90 and CD105, and poorly for LNGFR, STRO1, CD34 and CD56. Occasional cells stained positively for 3G5 from both the synovial fat pad and bone marrow. No staining was observed for the IgG control. 
growth factor mediated signaling pathways have been shown to induce or regulate the differentiation of MSCs along mesenchymal lineages as Bone morphogenic protiens (BMP) and fibroblast growth factor (FGF) are examples for these growth factors [15].

\section{WHY STEM CELLS ARE NOT IMMUNOGENIC?}

Ultimately, the immunology of MSCs, similar to many other aspects of these cells, remains poorly defined. Welldesigned in vivo studies of the immunogenicity and the immune-modulatory capacity of MSCs in specific pathophysiologic models are needed before further claims regarding the immune properties of MSCs can be made. The known immunologic properties of MSCs enhance their therapeutic appeal. However, as with most analysis of MSCs, these immune properties have been observed exclusively on cultured cells. The immune phenotype of cultured MSCs is widely described as MHC Class I +ve, MHC Class II -ve, CD40 -ve, CD80 -ve, and CD86 -ve [16, 17].

This phenotype is regarded as non-immunogenic and suggests that MSCs might be effective in inducing tolerance. Class I may activate alloreactive $\mathrm{T}$ cells, but with the absence of co-stimulatory molecules, a secondary signal would not engage, leaving the T cells anergic [18]. MSCs are able to inhibit production of cytotoxic lymphocytes in vitro and resist killing by both cytotoxic lymphocytes and natural killer cells. Also MSCs are proved to inhibit peripheral blood mono-nuclear cells (PBMCs) even if external co-stimulatory molecules are added. This was explained presumably through soluble factors production [17]. Once again, there is a surprising lack of data on the immunomodulatory effects of MSCs in vivo. It was reported that MSCs have prolonged allogeneic skin graft survival in immunocompetent, outbred baboons [19].

\section{BONE TISSUE ENGINEERING}

Tissue engineering is an emerging interdisciplinary field that applies principles of both life sciences and engineering towards the development of biological substitutes that restore, maintain, and improve function of damaged and/or lost tissues $[3,20]$. Tissue engineering is now occupying front position in regenerative medicine field. Specially, after emerging of another class of tissue engineering which is the cell based tissue engineering; where structural and functional defects are completely restored. This achieves the superior aim of regenerative medicine by substitution of missed tissues with the same type of tissue [20]. The various types are described below.

\section{Local Recruitment of Osteogenic Cells}

An important progress in treating bone defects has been the introduction of bone morphogenetic proteins (BMPs) specifically BMP-2, BMP-4 and BMP-7. These proteins induce osteogenic cell differentiation in vitro, as well as bone defect healing in vivo. Carriers enhancing the binding of BMPs are of the most importance, since extended BMP release creates an osteogenic microenvironment that allows multipotent cell progenitors to migrate to the area of injury and to proliferate and differentiate toward the osteogenic lineage. The four major categories of BMP carrier materials are natural polymers, inorganic materials, synthetic polymers and composites of these materials [3].

This, however will fail if the vitality and the number of the patient's multipotent cells are insufficient or if the patient is aged and with a poor general health status. The last condition includes patients with large bone defects, who underwent radiation or chemotherapy, elderly, immunocompromized, malnourished patients and other. Milligrams of BMPs are problematic as regards manufacturing, cost and dose [1,3].

\section{Gene Therapy and Tissue Engineering}

MSCs have also shown great promise in the delivery of genes or gene products. The ability of MSCs to be transduced gives them the potential to serve as vehicles or either long-term or short-term therapeutic gene transfer, for instance, as in expressing BMP for bone repair. BMP transduced bone marrow derived stem cells have shown effective power in repair of critical sized defects in different animal models and different sites [21].

On the other hand, the administration of supraphysiological doses of BMP has been shown to induce the opposite effect by stimulating bone resorption and turnover, yielding a counter productive response. Therefore, utilizing MSCs as a BMP delivery vehicle may ensure the sitespecific administration of physiological doses of BMP [22].

\section{In Vitro Osteoinduction of Stem Cells}

Osteoinductive cytokines is added to the routine culture media in order to control stem cells differentiation towards osteoblast lineage. Among the most popular induction protocols is the combination of $\beta$-glycerophosphate, dexamethasone and ascorbic acid. Where $\beta$-glycerophosphate is a source for organic phosphate, dexamethasone is crucial in expression of osteogenic phenotype in certain concentrations, and ascorbic acid important in collagen and mineral deposition. Other osteoinductive growth factors and cytokines include transforming growth factor $\beta$ (TGF- $\beta_{1}$ ), fibroblast growth factor (FGF), active vitamin D, BMPs (BMP 2, 4 and 7) and parathyroid hormone [13].

It is known that osteoblastic differentiation from undifferentiated state to functional active osteoblast is a series of steps involving a number of proteins expressed at each stage, such as Alkaline Phosphatase (ALP), osteocalcin and mineral deposition. In general, ALP is considered as a relatively early marker of osteoblast differentiation. During the bone formation, the extracellular matrix progressively assumes bone-like properties and then, with the onset of mineralization, there is an increased activity of noncollagenous extracellular matrix protein, such as osteocalcin, and deposition of inorganic crystals composed of calcium and phosphate. At the end, loading of a suitable scaffold with induced cells hand in hand with incubation of the whole culture in bioreactor provide efficient but expensive way to generate bone [12].

\section{Ex-Vivo Production of Tissue Engineered Bone}

The stem cell-based tissue engineering of bone includes three main steps. First, stem cell harvesting, isolation and expansion. Secondly, scaffold seeding with induced stem cells. Finally, re-implantation is done in vivo to reconstruct a 
defect. This field has received a great deal of attention owing to its affinity as an alternative to bone grafts and substitutes for reconstruction of bone [9].

\section{WHAT IS A SCAFFOLD?}

Stem cells are often implanted or seeded into a structure capable of supporting three-dimensional tissue formation. These structures, typically called scaffolds, are often critical, both ex vivo as well as in vivo, to recapitulating the in vivo milieu and allowing cells to influence their own microenvironments. Tissue engineering approaches to skeletal reconstruction evoke the general principles of using porous scaffolds that deliver biofactors (cells, genes and proteins) to regenerate natural tissue. Scaffolds must also meet the typical requirements for mechanically functioning tissues of enhancing tissue regeneration through biofactor delivery while maintaining temporary mechanical function until the tissue can bear load. However, in certain areas of reconstruction (e.g. craniofacial); scaffolds must fit very complex three-dimensional (3D) anatomic defects that can be much more complicated than those in the appendicular skeleton [23].

\section{BASIC REQUIREMENTS FOR SCAFFOLDS}

Scaffolds should fit complex 3D anatomic defects. This can be achieved roughly through surgical experience in designing or highly sophisticated computed designing systems [24]. They should provide temporary load bearing skeleton until neo-tissue forms. Biodegradability is often an essential factor since scaffolds should preferably be absorbed by the surrounding tissues without the necessity of a surgical removal. The rate at which degradation occurs has to coincide as much as possible with the rate of tissue formation: this means that while cells are fabricating their own natural matrix structure around themselves, the scaffold is able to provide structural integrity within the body and eventually it will break down leaving the neo-tissue, newly formed tissue which will take over the mechanical load [25].

Scaffolds should enhance tissue regeneration through biofactor delivery. To achieve the goal of tissue reconstruction, scaffolds must be porous with adequate pore size; to facilitate cell seeding and diffusion throughout the whole structure of both cells and nutrients. Porosity ranged from $300-1200 \mu \mathrm{m}$ diameter is efficient in supporting cell migration, proliferation and growth factor transport. Smaller diameter porosity is not efficient while larger one can affect mechanical properties of the scaffold. So coupling of both, scaffold load bearing properties and porosity is of a paramount importance in having ideal scaffold. Both requirements are variable according to the area under the interest of reconstruction purposes [23].

\section{TYPES OF SCAFFOLDS}

Many different materials (natural and synthetic, biodegradable and permanent) have been investigated. Most of these materials have been known in the medical field before the advent of tissue engineering as a research topic, being already employed as bioresorbable sutures. Examples of these materials are collagen and some polyesters. New biomaterials have been engineered to have ideal properties and functional customization: injectability, synthetic manufacture, biocompatibility, non-immunogenicity, transparency, nano-scale fibers, resorption rates, etc [26].

There are broadly two groups of scaffolds and scaffolds from natural materials are the first group. In particular different derivatives of the extracellular matrix have been studied to evaluate their ability to support cell growth. Proteic materials, such as collagen or fibrin, and polysaccharidic materials, like chitosan or glycosaminoglycans (GAGs), have all proved suitable in terms of cell compatibility, but some issues with potential immunogenicity still remains. Among GAGs, hyaluronic acid, possibly in combination with cross linking agents (e.g. glutaraldehyde, water soluble carbodiimide etc), is one of the possible choices as scaffold material [26]. Also placental decellular matrix (PDM) was evaluated by Flynn and his coworkers as regards its effect on seeded cells activity [25]. Natural coral with a pore size of 150 to $220 \mu \mathrm{m}$ and porosity of about $36 \%$ was molded into the shape of a human mandibular condyle. This Coral gives significant results as a scaffold in addition to recombinant human bone morphogenetic protein-2 (rhBMP-2) [24].

The second group is synthetic scaffolds, and a commonly used synthetic material is PLA- polylactic acid. This polyester which degrades within the human body to form lactic acid, a naturally occurring chemical which is easily removed from the body. Similar materials are polyglycolic acid (PGA) and polycaprolactone (PCL), their degradation mechanism is similar to that of PLA, but they exhibit respectively a faster and a slower rate of degradation compared to PLA [26]. Researches aim at creating scaffold materials with biomimetic properties that would mimic the role of the extracellular matrix (ECM) in many cell functions, including: adhesion, migration. Further engineering of biomaterial surface and bulk properties will allow bio-specific interactions between appropriate cell types and scaffold materials and proliferation. Porosity is one of the essentials for successful scaffolding materials. A number of different methods have been described in literature for preparing porous structures to be employed as tissue engineering scaffolds. Each of these techniques presents its own advantages, but none is devoid of drawbacks [26].

\section{DISCUSSION}

The Craniofacial, Maxillofacial, upper and lower limb reconstructive procedures often require bone graft harvesting, which results in donor site morbidity. The Cell based tissue engineering is the cornerstone technique in tissue regeneration; especially in bone production. Bone tissue engineering overcomes the drawbacks of the common techniques of bone reconstruction [6].

Bone tissue engineering is considered as an unlimited source of bone production without donor site morbidity and other autogenous bone grafting restrictions. There is no risk of infection transmission or rejection as may occur with demineralised bone derived from human cadavers or animal sources. Also it gives us a compatible material without any rejection or immune reaction against it. So it covers the cases where alloplastic materials are contraindicated because of rejection or growth restrictions [7].

It has been established that critical size cranial defects have been widely used as a model to measure the efficiency 
of different bone repair techniques. Owing to the fact that there is less blood supply, less marrow and thick cortical bone in the cranium; so it is hard to repair calvarial bone defects. Many studies were done on the critical size cranial defects of mouse, rat, rabbit, and other animals [27].

A lot of studies were done to evaluate, standardize and justify the optimal technique for bone tissue engineering. In China Fulin 2002 and his colleagues [24] studied the use of bone marrow derived MSCs seeded coral scaffold in reconstruction of mandibular condylar defects in nude mice model; where recombinant human bone morphogenetic protein-2 (rhBMP2) is used to improve osteoblastic phenotype of cultured cells. Successful results were obtained after 2 months where the reconstructed bone retained the coral shape, which was manipulated to resemble mandibular condyle. The use of donor rabbit gives the advantage of having an unlimited source for stem cells, but the use of nude mice was mandatory to avoid immune reaction as was believed.

In 2004, Abukawa et al. [28] evaluated the use of autologous bone marrow stem cells to reconstruct mandibular defects. Osteoinduction of the stem cells was done by the use of $\beta$-glycerophosphate, dexamethazone and ascorbic acid (standard osteo-induction cytokines). This study introduced the use of poly-DL-lactic coglycolic acid polymer as a biodegradable scaffold that can support tissues until new bone was produced. Ever since, biodegradable synthetic polyesters were considered as the standard scaffolding material in the process of bone tissue engineering. Polyesters are completely absorbed after an interval of time. This interval is enough for newly formed bone to take the function of tissue support. Also polyesters can be manipulated as regards the physical properties like shape, surface area and porosity.

In 2006, Mankani et al. [29] used autologous bone marrow stem cells in higher animal models to ensure the efficiency of bone tissue engineering techniques to reconstruct sizable bony defects. Osteo-induction was done by the standard osteo-induction cytokines. Calcium phosphate ceramics were used as scaffold. These ceramics were non-biodegradable materials, which can interfere with the mechanical properties of the produced bone. This was explained by the fact that the resultant tissue was a combination of bone and calcium phosphate ceramics; which makes this tissue more fragile than the natural pure bone.

They evaluated the characters of the produced bone in vivo through ultrasonic examination which showed more bone formation than the control areas. Also he commented on the mechanical properties of the produced bones which were more than the control. Yet he did not compare the mechanical properties of the produced bones with the normal skull bones; mechanical properties were examined in ex-vivo fashion.

Once again Hou et al. [27] evaluated autologous bone marrow derived MSCs seeded corals with added rhBMP2 in reconstruction of New Zealand rabbit calvarial bone defects. They compared the results with other equivalent groups reconstructed with (1) autologous iliac cortico-cancellous bone graft, (2) corals with rhBMP2, (3) rhBMP2 only. The produced tissue was evaluated clinically, radiologically and histologically. Autologous bone grafts gave the highest success rate $(84 \%)$ with best quality for the produced bone. Bone tissue engineering (corals +induced stem cells + rhBMP2) gave comparable results with autologous bone graft $(77.9 \%)$. Coral and rhBMP2, and coral only groups gave the least results with higher results to the former group.

Bone tissue engineering studies have never used allograft of MSCs for reconstruction of bone defects. But several studies were done in other fields to study the effect of MSCs if used as allograft (studies for transplantation immunology). Tse and his coworkers [17] studied in vitro immune characteristics of human bone marrow derived MSCs. This study built up the principles of understanding the immune modulating action of MSCs.

Basically, MSCs' phenotype was determined as being MHC class I positive and negative for MHC class II; except if treated with gamma interferon. MSCs are negative for other co-stimulatory antigens (CD80, CD86 and CD40). Tse and his coworkers 2003 [17] concluded that this phenotype leaves the peripheral blood mononuclear cells anergic with no proliferative properties against MSCs or other allogenic cells in presence of MSCs. They assumed that this inhibitory effect of MSCs isn't only through direct cellular contact but rather through produced diffusible inhibitory factors. This was proved by culturing of stimulated proliferating mononuclear cells in the same culture with bone marrow derived MSCs. The proliferation of the activated mononuclear cells was inhibited by the presence of stem cells; in spite of the presence of semi-permeable membrane in between the two cell populations.

Silva et al. [30] used allograft of MSCs in treatment of animal chronic myocardium ischemic model. Results of this study showed the effectiveness of this treatment modality and commented on the allogenic properties of the stem cells used. Surprisingly, the stem cells were completely differentiated into vascular endothelial cells and smooth muscle cells; where they were successfully engrafted into the neo-vasculature induced in the ischemic myocardium. This successful engraftment resulted in improvement of cardiac vascularity and contractility. No immune suppression drugs were used in this study.

Matsumoto et al. [14] went through a more advanced study. They used acute myocardium ischemic animal model, where bone marrow derived MSCs were transfected with vascular endothelial growth factor (VEGF) gene through adenovirus vector. These cells were cultured and showed progressive increase in the levels of VEGF in the culture media. After in vivo injection of these cells inside the acutely ischemic hearts models, all experimental animals showed improvement of the cardiac vascularity and contractility. Radioactive isotope tracing showed that stem cells were engrafted successfully in the angiogenesis process and they were differentiated in endothelial cells with active production of VEGF.

\section{CONCLUSION}

Basically, autogenous bone graft is the standard technique in reconstruction of bone defects. All alternative techniques are aiming to avoid donor site morbidity and other complications. Recently bone tissue engineering offers a superior technique in management of bone defects. Bone 
tissue engineering is achieved through three main steps: (1) Stem cell harvesting, isolation and expansion. (2) Scaffold seeding with osteo-induced stem cells. (3) In vivo reimplantation.

Adult MSCs of bone marrow or adipose tissue origin are the standard sources for stem cells used in bone tissue engineering. On the other hand, scaffold choice is still under investigation in order to be standardized. So bone tissue engineering has all the advantages of autogenous bone grafting but with no donor site morbidity. Also it can be considered as unlimited source of allografts for bone reconstruction; as the non immunogenicity character of stem cells advocates the idea of stem cells banking. These banked cells could be used as an immediate unlimited source for bone reconstruction.

In conclusion, using bone marrow derived MSCs proved to be an efficient technique for bone reconstruction. In spite of being allogenic, stem cells were used to manage the problematic bone defects.

\section{ACKNOWLEDGEMENT}

None declared.

\section{CONFLICT OF INTEREST}

None declared.

\section{REFERENCES}

[1] Hollinger JO, Winn SR, Sfeir C and Schmidz JP. Bone regeneration options for craniofacial skeleton. In: Craniofacial surgery, Ed. Kant Y. Lin, Roy C. Ogle and John A. Jane. Philadelphia: WB. Saunders 2002; 113-29.

[2] Boutros SG. Structural support: cartilage and bone, In: Current therapy in plastic surgery. Joseph G M, Robert DG, Sean G B, Ed. Philadelphia: WB. Saunders 2006; 3: 22-8.

[3] Shanti RM, Li WJ, Nesti LJ, Wang X, Tuan RS. Adult mesenchymal stem cells: Biological properties, Characteristics, and Surgical maxillofacial Applications. J Oral Maxillofac Surg 2007; 65:1640-47.

[4] Pritsch T, Bickels J, Wu CC, Squires HM, Malawer MM. The risk for fractures after curettage and cryosurgery around the knee. Clin Orthop Relat Res 2007; 1:59-6.

[5] Muramatsu K, Doi K, Ihara K, Shigetomi M, Kawai S. Recalcitrant posttraumatic nonunion of the humerus: 23 patients reconstructed with vascularized bone graft. Acta Orthop Scand 2003; 1: 95-7. Quoted from: Cancedda R, Paolo G. Maddalena M. Review, A tissue engineering approach to bone repair in large animal models and in clinical practice. Biomaterials 2007; 28: 4240-50.

[6] Chou LB, Mann RA, Coughlin MJ, McPeake III WT, Mizel MS. Stress fracture as a complication of autogenous bone graft harvesting from the distal tibia. Foot Ankle Int 2007; 2: 199-201.

[7] Mroz TE, Lin EL, Summit MC, Bianchi JR, Keesling Jr JE, Roberts M. Biomechanical analysis of allograft bone treated with a novel tissue sterilization process. Spine J 2006; 1: 34-9.

[8] Marcacci M, Kon E, Zaffagnini S, Giardino R, Rocca M, Corsi A. Reconstruction of extensive long-bone defects in sheep using porous hydroxyapatite sponges. Calcif Tissue Int 1999; 1: 83-90.

[9] Cancedda R, Paolo G, Maddalena M. Review, A tissue engineering approach to bone repair in large animal models and in clinical practice. Biomaterials 2007; 28:4240-50.
[10] Lendeckel S, Jodicke A, Christophis P. Autologous stem cells (adipose) and fibrin glue used to treat widespread traumatic calvarial defects: Case report. J Craniomaxillofac Surg 2004; 32: 370.

[11] Svendsen C. Stem cells: Hype or hope? Drug Discov Today 2002; 7: 455 .

[12] Park BW, Hah YS, Kim DR, Kim JR, Byun JH. Osteogenic phenotypes and mineralization of cultured human periostealderived cells. Arch Oral Biol 2007; 52: 983-89.

[13] Zuk PA, Sasano Y, Takahashi I, Mizoguchi I, Kagayama M. Multilineage cells from human adipose tissue. Tissue Eng 2001; 7: 211-28.

[14] Matsumoto R, Omura T, Yoshiyama M, et al. Transplantation for the treatment of acute myocardial infarction vascular endothelial growth factor-expressing mesenchymal stem cell. Arterioscler Thromb Vasc Biol 2005; 25: 1168-73.

[15] Tuan RS, Boland G, Tuli R. Adult mesenchymal stem cells and cell-based tissue engineering, Arthritis Res Ther 2003; 5: 32 .

[16] Le Blanc K, Tammik C, Rosendahl K, Zetterberg E, Ringden O. HLA expression and immunologic properties of differentiated and undifferentiated mesenchymal stem cells. Exp Hematol 2003; 31: 890-96.

[17] Tse WT, Pendleton JD, Beyer WM, Egalka MC, Guinan EC Suppression of allogeneic T-cell proliferation by human marrow stromal cells: implications in transplantation. Transplantation 2003; 75: 389-97

[18] Le Blanc K, Tammik L, Sundberg B, Haynesworth SE, Ringden O. Mesenchymal stem cells inhibit and stimulate mixed lymphocyte cultures and mitogenic responses independently of the major histocompatibility complex. Scand J Immunol 2003; 57: 11-20.

[19] Javazon EH, Beggs KJ, Flake AW. Beggs, AW. Flake. Mesenchymal stem cells: Paradoxes of passaging. Exp Hematol 2004; 32: 414-25.

[20] Kneser U, Schaefer DJ, Polykandriotis E, Horch RE. Tissue engineering of bone: The Reconstructive surgeon's point of view. J Cell Mol Med 2006; 10: 7-19.

[21] Chang SC, Wei FC, Chuang H, et al. Ex vivo gene therapy in autologous critical-size craniofacial bone regeneration. Plast Reconstr Surg 2003; 112: 1841.

[22] Nakashima M, Reddi AH. The application of bone morphogenetic proteins to dental tissue engineering. Nat Biotechnol 2003; 21: 1025.

[23] Hollister SJ, Lin CY, Saito E, et al. Engineering craniofacial scaffolds. Orthod Craniofacial Res 2005; 8: 162-73.

[24] Chen F, Mao T, Tao K, Chen S, Ding G, Gu X. Bone Graft in the Shape of Human Mandibular Condyle Reconstruction via Seeding Marrow-Derived Osteoblasts Into Porous Coral in a Nude Mice Model. J Oral Maxillofac Surg 2002; 60: 1155-9.

[25] Flynn L, Prestwich GD, Semple JL, Woodhouse KA. Adipose tissue engineering with naturally derived scaffolds and adiposederived stem cells. Biomaterials 2007; 28: 3834-42.

[26] Elisseff J, Ma PX. Scaffolding in tissue engineering. Boca Raton: CRC. E. Book, Downloaded at $15^{\text {th }}$ January 2009, ISBN 1m57444-521-9, 2005.

[27] Hou R, Chen F, Yang Y, et al. Comparative study between coralmesenchymal stem Cells rhBMP-2 composite and auto-bone-graft in rabbit critical-sized cranial defect model. Journal of Biomedical Materials Research Part A 2006; 5: 87-99.

[28] Abukawa H, Shin M, Williams WB, Vacanti JP, Kaban LB, Troulis MJ. Reconstruction of Mandibular Defects With Autologous Tissue-Engineered Bone. J Oral Maxillofac Surg 2004; 62: 601-6.

[29] Mankani MH, Kuznetsov SA, Shannon B, et al. Canine Cranial Reconstruction Using Autologous Bone Marrow Stromal Cells. Am J Pathol 2006; 168: 542-50.

[30] Silva GV, Litovsky S, Assad JA, et al. Mesenchymal stem cells differentiate into an endothelial phenotype, enhance vascular density, and improve heart function in a canine chronic ischemia model. Circulation 2005; 111: 150-156. 